\title{
Chronologic aging decreases tumor angiogenesis and metastasis in a mouse model of head and neck cancer
}

\author{
BOJANA BOJOVIC and DAVID L. CROWE \\ University of Illinois Cancer Center, University of Illinois at Chicago, \\ 801 S. Paulina St., Room 530C, MC860, Chicago, IL 60612, USA
}

Received November 2, 2009; Accepted December 28, 2009

DOI: 10.3892/ijo_00000547

\begin{abstract}
The incidence of malignant tumors increases with age. This may be due to the duration of carcinogenesis or age related changes providing a favorable environment for tumor formation. Aging is associated with molecular, cellular and physiological events that influence carcinogenesis and cancer growth. Physiologic cell proliferation, differentiation, and aging can result in cell death. However, under the influence of exogenous or endogenous factors cells can undergo pathologic dedifferentiation, immortalization, and neoplastic clone formation. The effects of age have been recognized in both animal and human malignancies. These processes can result in cellular senescence as a barrier to tumorigenesis. Inducing senescence is an important outcome for the successful treatment of cancers particularly those resistant to apoptosis. Senescence is associated with polyploidy in several human cell lines. Polyploid cells are dangerous in that they can undergo aberrant mitoses giving rise to unstable progeny. Polyploid cells have been shown to escape senescence and divide. We examined the effects of aging on squamous cell carcinoma formation in a mouse model. Chronologically aged mice experience shorter tumor latency periods than wildtype animals. Tumors in aged mice were poorly vascularized, necrotic, and produced significantly fewer cervical lymph node metastases. Vascular endothelial growth factor expression was similar in primary tumors from young and old mice, but microvessel density was significantly reduced in tumors arising in aged mice. These results indicate that host response to angiogenic factors inhibit tumor growth and metastasis of head and neck cancer.
\end{abstract}

\section{Introduction}

The incidence of malignant tumors increases with age (1). This may be due to the duration of carcinogenesis or age related changes providing a favorable environment for tumor

Correspondence to: Dr David L. Crowe, University of Illinois Cancer Center, University of Illinois at Chicago, 801 S. Paulina St., Room 530C, MC860, Chicago, IL 60612, USA

E-mail: dlcrowe@uic.edu

Key words: vascular endothelial growth factor, microarray, angiogenesis, cell cycle, necrosis formation (2). Aging is associated with molecular, cellular and physiological events that influence carcinogenesis and cancer growth (3). Physiologic cell proliferation, differentiation, and aging can result in cell death (4). However, under the influence of exogenous or endogenous factors cells can undergo pathologic dedifferentiation, immortalization, and neoplastic clone formation (5). The majority of tumors are caused by environmental and lifestyle related factors (6). There is similarity between aging and carcinogenesis via systemic regulation at the physiologic level. In this regard, immunosuppression is a common feature of aging and carcinogenesis (7). Dysfunctional T lymphocytes have been characterized in elderly humans (8). Alterations of immune surveillance may contribute to age associated cancer. Decreased specific T cell responses to tumor antigens may explain this phenomenon (9).

The effects of age have been recognized in both animal and human malignancies (10). DNA damage is believed to be high in human cells. Mechanisms of DNA repair have evolved to deal with DNA damage. These processes can result in cellular senescence as a barrier to tumorigenesis (11). Many tumor cells have the ability to undergo senescence in vitro (12). Cancer cells exposed to DNA damaging agents undergo permanent cell cycle arrest and acquire a phenotype similar to that observed in senescence of normal human cells (13). Senescent cells have been reported in breast cancer biopsies and those from lung cancer patients receiving chemotherapy $(14,15)$. Inducing senescence is an important outcome for the successful treatment of cancers particularly those resistant to apoptosis (16). Cancer cells can be blocked in G2 or bypass cell cycle checkpoints and undergo endo-reduplication. Senescence is associated with polyploidy in several human cell lines (17). Polyploid cells are dangerous in that they can undergo aberrant mitoses giving rise to unstable progeny (18). Polyploid cells have been shown to escape senescence and divide (19).

To determine the effects of aging on a mouse head and neck cancer model (20), we compared the phenotypes of chemically induced head and neck cancer in mice that began the tumor induction protocol at 1 month and 1 year of age. We determined that aging decreases vascularization and tumor metastasis in the murine model.

\section{Materials and methods}

Mouse procedures. This study was approved by the institutional animal care and use committee before any experiments 
were performed. C57B16J mice were used at 1 month and 1 year of age. Mice were housed in approved environmentally controlled facilities on $12 \mathrm{~h}$ light-dark cycles with unlimited access to food and water. Twenty male and female 1 month old and 1 year old mice were dosed orally twice weekly using $25 \mu \mathrm{l}$ dimethylbenzanthracene (DMBA) dissolved in $20 \mu \mathrm{l}$ ethanol. The time course and number of tumors were recorded for each animal. Mice were euthanized when any institutional criterion for experimental neoplasia in rodents was met. Euthanized mice were photographed and complete necropsies were performed. A portion of each tumor specimen was flash frozen in liquid nitrogen or fixed in $4 \%$ buffered formaldehyde for $16 \mathrm{~h}$ at room temperature.

Histopathology and immunohistochemistry. Tumor tissue was dehydrated in an ethanol series, cleared in xylene, and embedded in paraffin. Five micrometer sections were prepared and mounted on poly-L-lysine coated slides. Representative sections were stained with hematoxylin and eosin and histologically evaluated by a pathologist. Immunohistochemical analysis was performed using a commercially available kit (Invitrogen, Carlsbad, CA). Sections were incubated at $60^{\circ} \mathrm{C}$ for $30 \mathrm{~min}$ and deparaffinized in xylene. Endogenous peroxidase activity was quenched by incubation in a 9:1 methanol/ $30 \%$ hydrogen peroxide solution for $10 \mathrm{~min}$ at room temperature. Sections were rehydrated in PBS ( $\mathrm{pH} 7.4$ ) for $10 \mathrm{~min}$ at room temperature. Sections were blocked with $10 \%$ normal serum for $10 \mathrm{~min}$ at room temperature followed by incubation with anti-TGF $\alpha, \mathrm{HGF}$, c-met, cyclin A, cyclin D, cyclin E, c-myc, PCNA, and VE-cadherin antibodies (Santa Cruz Biotechnology, Santa Cruz, CA) for $16 \mathrm{~h}$ at room temperature. After three washes in PBS, the sections were incubated with secondary antibody conjugated to biotin for $10 \mathrm{~min}$ at room temperature. After additional washes in PBS, the sections were incubated with streptavidin conjugated horseradish peroxidase for $10 \mathrm{~min}$ at room temperature. Following final washes in PBS, antigen-antibody complexes were detected by incubation with hydrogen peroxide substrate solution containing aminoethylcarbazole chromogen reagent. Slides were rinsed in distilled water, coverslipped using aqueous mounting medium, and allowed to dry at room temperature. The relative intensities of the completed immunohistochemical reactions were evaluated using light microscopy by independent trained observers who were unaware of the mouse genotypes. A scale of 0 to 3 was used to score relative intensity, with 0 corresponding to no detectable immunoreactivity and 1, 2 and 3 equivalent to low, moderate, and high expression, respectively. The number of capillaries per square millimeter in SCCs from each group were counted. Non-parametric data were analyzed by Fisher's exact test.

RNA extraction and gene expression profiling. Total RNA was extracted from microdissected primary and metastatic tumor tissue using a commercially available kit (RNEasy, Qiagen, Valencia, CA). VEGF expression was analyzed by quantitative RT-PCR using primers 5'-GGAAAGGGAAAGG GTCAAAAAC-3' and 5'-TTCTCACATCTGCAAGTACG-3' in $20 \mathrm{mM}$ Tris- $\mathrm{HCl}$ ( $\mathrm{pH} \mathrm{8.3),} 1.5 \mathrm{mM} \mathrm{MgCl}_{2}, 63 \mathrm{mM} \mathrm{KCl}$, $0.05 \%$ Tween-20, $1 \mathrm{mM}$ EGTA, $50 \mu \mathrm{M}$ of each dNTP, and $2.5 \mathrm{U}$ Taq DNA polymerase (Roche Applied Science).
Amplification with 3 -actin cDNA using primers 5 '- $-3^{\prime}$ and 5 '- -3 ' as the internal control was carried out by real-time PCR (iCycler, Bio-Rad) using SYBR Green and cycle parameters $94^{\circ} \mathrm{C}$ for $25 \mathrm{sec}, 55^{\circ} \mathrm{C}$ for $1 \mathrm{~min}$, and $72^{\circ} \mathrm{C}$ for $1 \mathrm{~min}$.

Individually matched well differentiated primary and metastatic tumor tissue was used in microarray analysis. Three independent samples from each group were used in gene expression analysis. The integrity of rRNA bands was confirmed by Northern gel electrophoresis. Total RNA $(10 \mu \mathrm{g})$ with spike in controls was reverse transcribed using a T7oligo(dT) promoter primer in the first strand cDNA synthesis reaction. Following RNase $\mathrm{H}$ mediated second strand synthesis, the double stranded cDNA was purified and served as template in the subsequent in vitro transcription reaction. The in vitro transcription reaction was carried out in the presence of $\mathrm{T} 7$ RNA polymerase and a biotinylated nucleotide analogue/ ribonucleotide mix for complementary RNA amplification and biotin labeling. The biotinylated complementary RNA targets were then purified, fragmented, and hybridized to Affymetrix GeneChip Expression arrays (Santa Clara, CA). The murine genome 4302.0 microarray was used to interrogate 39,000 possible transcripts in each sample. After washing, hybridization signals were detected using streptavidin conjugated phycoerythrin. Affymetrix GCOS software was used to generate raw gene expression scores and normalized to the relative hybridization signal from each experiment. All gene expression scores were set to a minimum value of 2 times background determined by GCOS software in order to minimize noise associated with less robust measurements of rare transcripts. Normalized gene expression data were imported into dChip software for hierarchical clustering analysis using the average linkage algorithm. Raw data were analyzed for quality control and the significance of differential gene expression determined by t-test $(\mathrm{p}<0.05)$ and ratio analysis (>2-fold)

\section{Results}

We characterized HNSCC in wild-type mice when the carcinogenesis protocol was begun at 1 month or 1 year of age. As shown in Fig. 1, primary HNSCC in the 1 year old group arose at significantly earlier time points on average compared to wild-type mice (18 weeks for the 1 year old mice vs. 22 weeks for 1 month old animals; $p<0.03$ ). These data indicate that primary HNSCC in chronologically aged mice develops with significantly decreased latency periods. Chronologically aged mice also demonstrated significantly fewer metastatic lymph nodes per mouse ( 1.8 positive nodes in the 1 year old group vs. 4.1 nodes in the 1 month old group; $\mathrm{p}<0.001$; Fig. 2). These results indicate that SCCs in chronologically aged mice are less metastatic than those in young mice.

Primary HNSCC in wild-type mice which began the induction protocol at 1 year old were much less vascularized (Fig. 3B) than those in young mice (Fig. 3A). The histopathologic appearance of HNSCC in chronologically aged mice was dramatically different from younger animals. While the percentage of well vs. moderately differentiated tumors was similar to that observed in younger mice, areas of necrosis were noted in the primary tumors which correlated with the less vascularized appearance of these cancers in vivo 


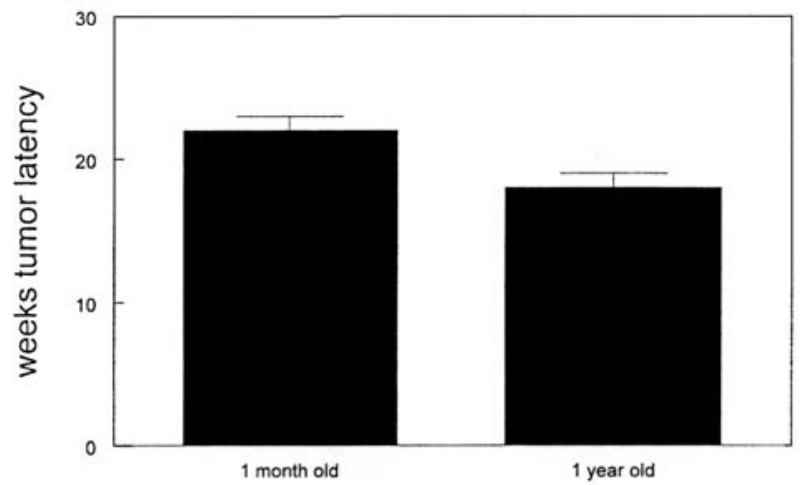

Figure 1. Decreased tumor latency in chronologically aged mice. The number of weeks to clinical tumor for mice that began the induction protocol at 1 month of age and those that began the protocol at 1 year of age was recorded. Error bars indicate SEM. These experiments were performed three times with similar results.

(Fig. 3C and D). Necrotic tumor tissue was also observed in metastatic lymph nodes in chronologically aged mice (Fig. 3E). These results indicate that both primary and metastatic HNSCC in chronologically aged mice are less vascularized resulting in necrotic cell death.

We examined expression of cell cycle regulatory proteins in SCC from young and chronologically aged mice by immunohistochemistry. Representative sections are shown in Fig. 4. Only $10 \%(2 / 21)$ of primary HNSCC from chrono-

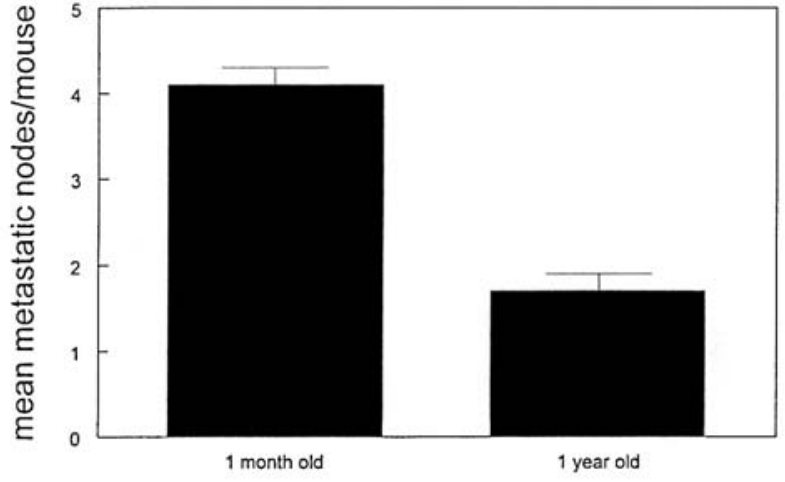

Figure 2. Decreased lymph node metastasis in chronologically aged mice. The number of histopathologically positive cervical lymph nodes in each tumor bearing mouse which began that protocol at 1 month versus 1 year of age was recorded. Error bars indicate SEM. These experiments were performed three times with similar results. logically aged mice overexpressed TGF $\alpha$ (compared to $89 \%$ in young mice), EGFR (39\% of young mice), cyclin A (56\% in young mice), or c-myc. Cyclin E was overexpressed in $30 \%$ $(6 / 21)$ of primary tumors in chronologically aged mice which was similar to that observed in young mice. Cyclin D was overexpressed in $33 \%$ (7/21) of primary SCC in chronologically aged mice compared to $44 \%$ in young mice. c-met was overexpressed in $48 \%(10 / 21)$ of primary tumors from chronologically aged mice. p16 expression was lost in $90 \%$
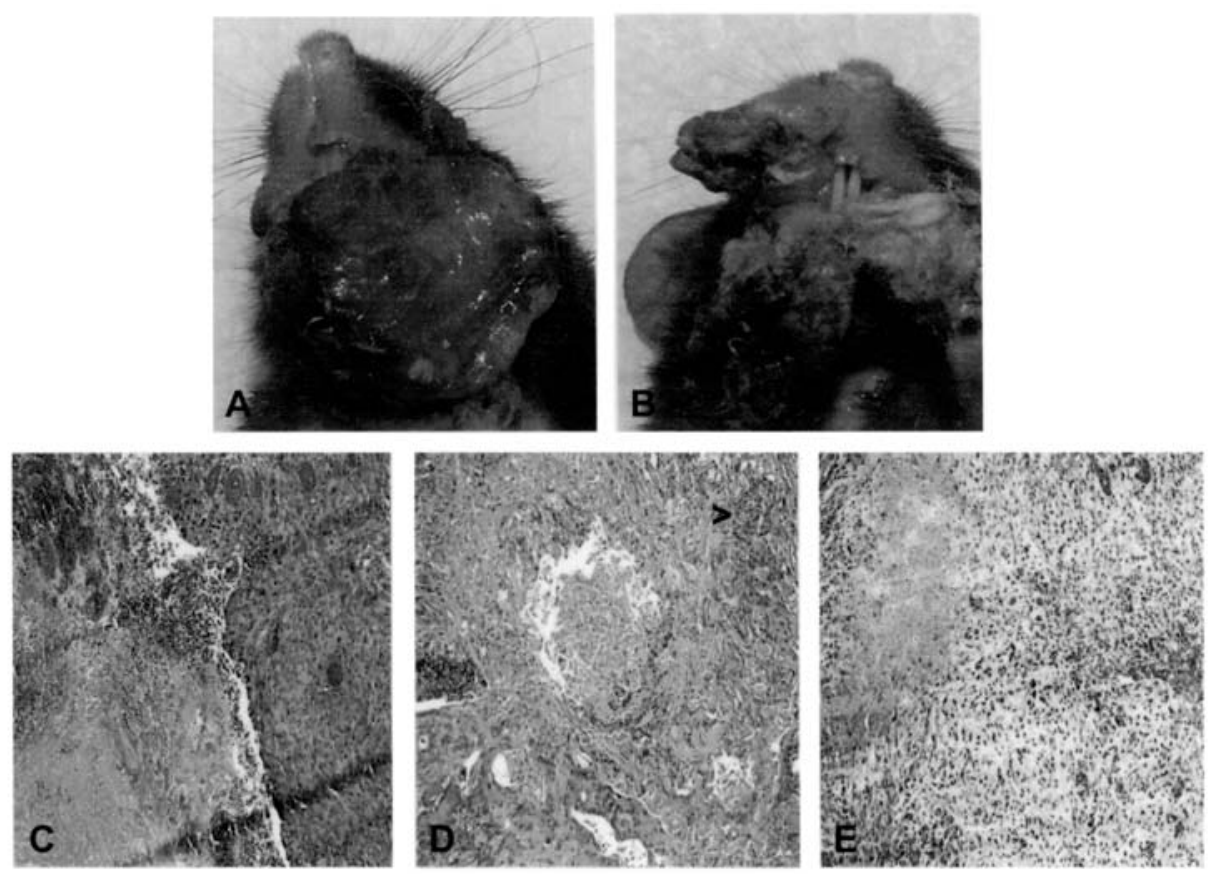

Figure 3. Necrosis in primary tumors of chronologically aged mice. (A) Representative gross appearance of late stage primary HNSCC in young and chronologically aged animals. Note high degree of vascularization of tumors in young mice. (B) Gross appearance of late stage primary HNSCC in chronologically aged mice. Note lack of vascularization of these tumors. (C) SCC in chronologically aged mice. Tumor cells are shown at right and a large area of necrosis is seen at left. Magnification, $x$ 40. (D) A large area of necrosis characteristic of SCC in chronologically aged mice is noted in the center of the section. Magnification, x40. (E) Metastatic SCC in a cervical lymph node from chronologically aged mice. A large area of necrosis is seen on the left. Magnification, $\mathrm{x} 40$. 

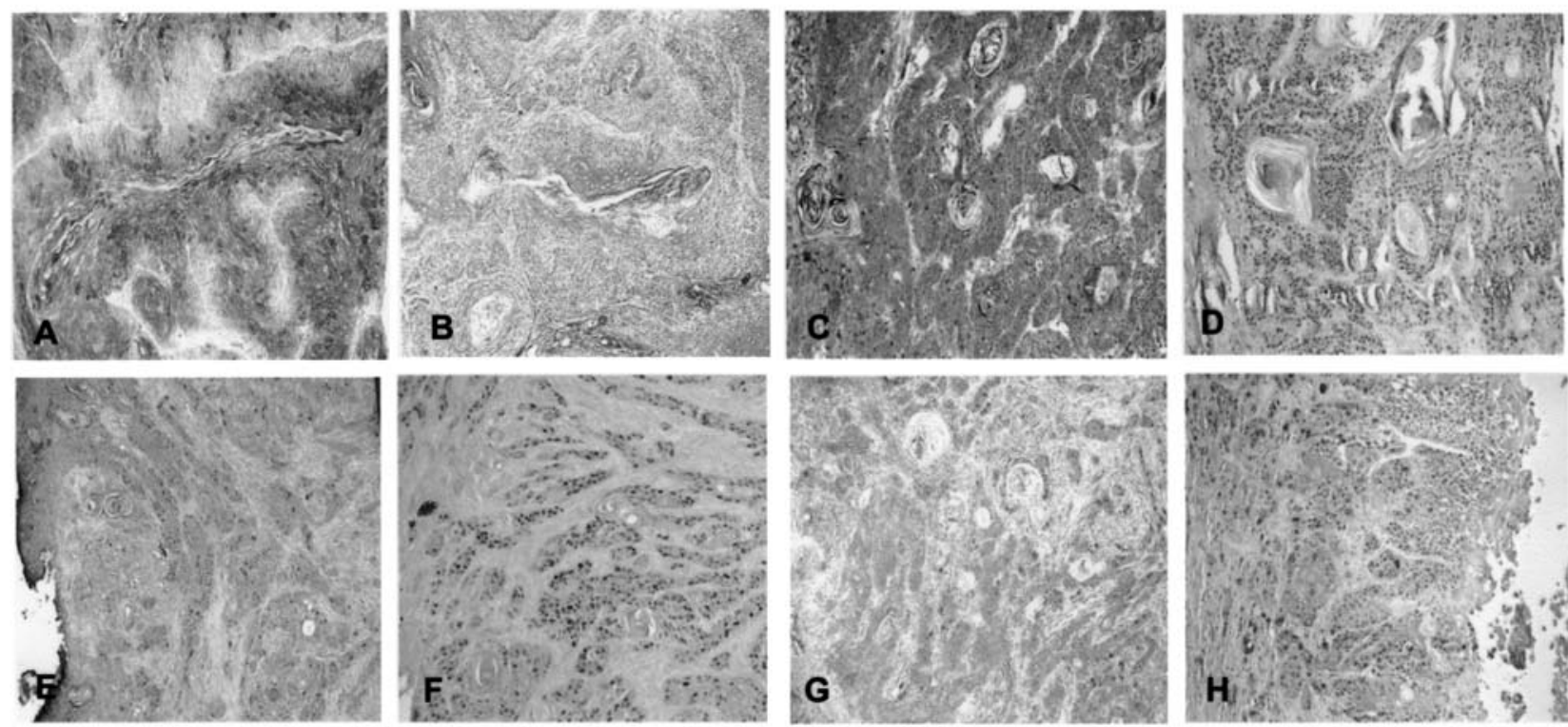

Figure 4. Expression of cell cycle regulatory proteins in SCC from chronologically aged mice. Tumor sections were analyzed by immunohistochemistry for expression of TGFa (A), HGF (B), c-met (C), PCNA (D), cyclin A (E), cyclin D (F), cyclin E (G), and c-myc (H). These experiments were performed 3 times with similar results. Representative sections are shown.

A

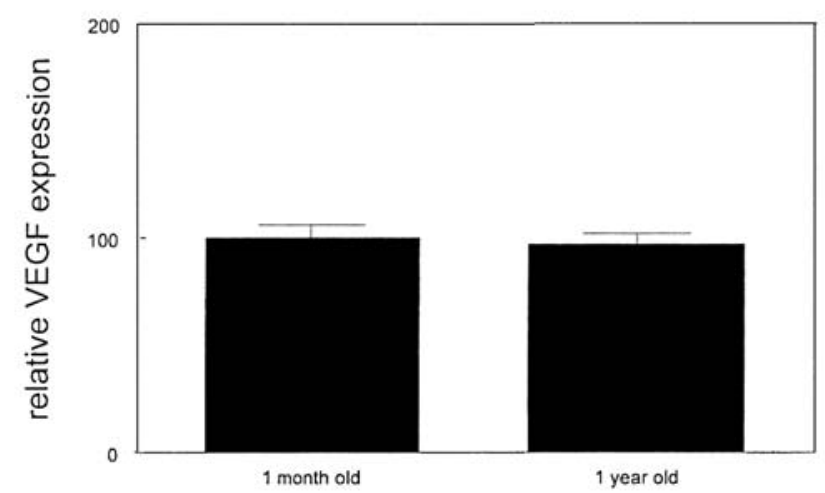

B

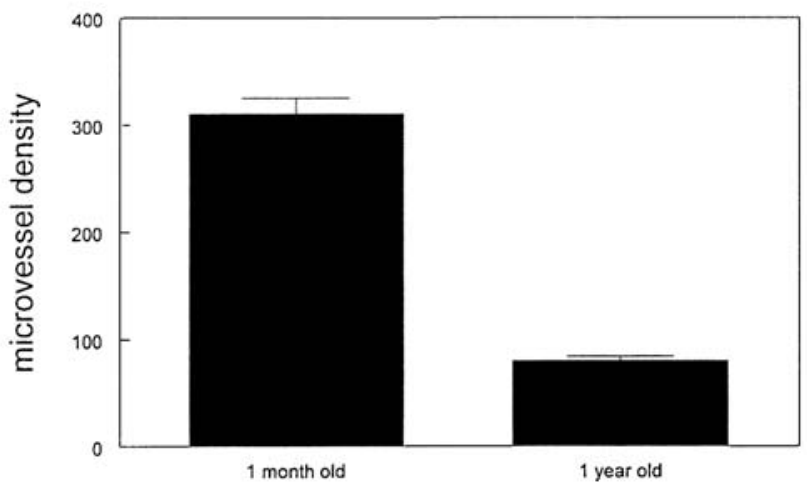

Figure 5. Reduced microvessel density in SCC from chronologically aged mice. (A) Similar VEGF mRNA expression in SCC from mice that began the tumor induction protocol at 1 month or 1 year of age is shown by quantitative RT-PCR. (B) The number of capillaries per square millimeter was counted following immunohistochemical staining with VE-cadherin. Error bars indicate SEM. These experiments were performed 3 times with similar results.

$(19 / 21)$ of primary tumors in chronologically aged mice. The number of PCNA positive (proliferating) cells was significantly decreased in SCC from chronologically aged mice $(30$ vs. $70 \%$ in young mice; $\mathrm{p}<0.01)$. In metastatic tumors from chronologically aged mice, EGFR was overexpressed in only $8 \%$ of cases (3/36). Cyclin A, cyclin B, cyclin E, and c-myc were not overexpressed in metastatic tumors from chronologically aged mice. These results indicate that while primary and metastatic HNSCC from young mice were similar to the human disease with respect to levels of cell cycle regulatory proteins, cancers from chronologically aged mice were dramatically different in expression of these genes.

To determine if decreased vascular endothelial growth factor (VEGF) expression by SCC could account for reduced vascularization of SCC in chronologically aged mice, we performed quantitative RT-PCR on mRNA extracted from tumors in young and aged mice. As shown in Fig. 5A, no significant differences in VEGF expression were observed between these tumors. These results indicate that changes in VEGF expression did not account for reduced vascularization of SCC in chronologically aged mice. However, microvessel density in chronologically aged tumors as determined by VEcadherin labeling was significantly less than those in young mice $\left(79 / \mathrm{mm}^{2}\right.$ vs. $311 / \mathrm{mm}^{2}$; Fig. $\left.5 \mathrm{~B}\right)$. These results indicate that capillary formation in chronologically aged mice was significantly less than in young mice, suggesting that the response to VEGF was decreased in older animals.

In tumors from chronologically aged mice, we microdissected tissue well away from areas of necrosis. We also used microarray analysis to determine differences between 
Table I. Gene expression changes between primary SCC in young and old mice (458 genes).

\begin{tabular}{|c|c|c|c|}
\hline Accession & Symbol & Gene name & Fold change \\
\hline NM_011478 & Sprr3 & Small proline-rich protein 3 & 31.0 \\
\hline NM_011146 & Pparg & Peroxisome proliferator activated receptor $\gamma$ & 26.7 \\
\hline U80011 & Pitx2 & Paired-like homeodomain transcription factor 2 & 18.0 \\
\hline NM_019728 & Defb4 & Defensin $B 4$ & 16.1 \\
\hline NM_007702 & Cidea & Cell death-inducing DNA fragmentation factor, $\alpha$ subunit A & 14.9 \\
\hline AF319173 & Psca & Prostate stem cell antigen & 14.6 \\
\hline NM_054074 & Defb6 & Defensin $\beta 6$ & 14.5 \\
\hline AV082644 & Krt4 & Keratin 4 & 13.2 \\
\hline Y11169 & Dsc3 & Desmocollin 3 & 10.9 \\
\hline NM_010662 & Krt13 & Keratin 13 & 9.8 \\
\hline BB410728 & E2f8 & E2F transcription factor 8 & 9.7 \\
\hline NM_013504 & Dsc1 & Desmocollin 1 & 7.6 \\
\hline NM_013505 & Dsc2 & Desmocollin 2 & 7.5 \\
\hline NM_012044 & Pla2g2e & Phospholipase A2, group IIE & 6.3 \\
\hline NM_015789 & Dkkl1 & Dickkopf-like 1 & 6.1 \\
\hline U65091 & Cited1 & $\mathrm{Cbp} / \mathrm{p} 300$-interacting transactivator & 6.0 \\
\hline AK007402 & Cgrrf1 & Cell growth regulator with ring finger domain 1 & 6.0 \\
\hline NM_008182 & Gsta1/Gsta2 & Glutathione S-transferase, a1/glutathione S-transferase, a2 & 5.9 \\
\hline BB211377 & Thsd4 & Thrombospondin, type I, domain containing 4 & 5.9 \\
\hline AK009343 & Serpina9 & Serine (or cysteine) peptidase inhibitor, clade A, member 9 & 5.9 \\
\hline AK014669 & Serpinb12 & Serine (or cysteine) peptidase inhibitor, clade B, member 12 & 5.7 \\
\hline BI150236 & Smad3 & MAD homolog 3 (Drosophila) & 5.6 \\
\hline AI172943 & Gsta3 & Glutathione S-transferase, $\alpha 3$ & 5.4 \\
\hline $\mathrm{U} 34245$ & Fosl1 & Fos-like antigen 1 & 5.3 \\
\hline AF169192 & Numb & Numb gene homolog (Drosophila) & 5.3 \\
\hline NM_009374 & $\operatorname{Tgm} 3$ & Transglutaminase 3 , E polypeptide & 5.1 \\
\hline BG916928 & Traf6 & Tnf receptor-associated factor 6 & 5.1 \\
\hline BI465650 & Ext1 & Exostoses (multiple) 1 & -5.0 \\
\hline ВC003806 & Stat3 & Signal transducer and activator of transcription 3 & -5.0 \\
\hline BI104444 & Cxc19 & Chemokine (C-X-C motif) ligand 9 & -5.1 \\
\hline BB475194 & Adamts5 & A disintegrin-like and metallopeptidase, thrombospondin motif, 5 & -5.2 \\
\hline AV221401 & Ephb2 & Eph receptor B2 & -5.2 \\
\hline NM_011658 & Twist1 & Twist gene homolog 1 (Drosophila) & -5.2 \\
\hline AA880220 & Jag1 & Jagged 1 & -5.6 \\
\hline BB454540 & Marcks & Myristoylated alanine rich protein kinase $\mathrm{C}$ substrate & -5.6 \\
\hline NM_013599 & Mmp9 & Matrix metallopeptidase 9 & -5.6 \\
\hline NM_018865 & Wisp1 & WNT1 inducible signaling pathway protein 1 & -5.6 \\
\hline M33760 & Fgfr1 & Fibroblast growth factor receptor 1 & -5.7 \\
\hline NM_021443 & $\mathrm{Ccl} 8$ & Chemokine (C-C motif) ligand 8 & -5.8 \\
\hline NM_133949 & Ptov1 & Prostate tumor overexpressed gene 1 & -5.8 \\
\hline NM_008809 & Pdgfrb & Platelet derived growth factor receptor, $\beta$ polypeptide & -6.0 \\
\hline NM_021274 & Cxcl10 & Chemokine (C-X-C motif) ligand 10 & -6.1 \\
\hline NM_016919 & Col5a3 & Procollagen, type V, $\alpha 3$ & -6.2 \\
\hline BM119402 & Ski & Sloan-Kettering viral oncogene homolog & -6.3 \\
\hline NM_008607 & Mmp13 & Matrix metallopeptidase 13 & -6.6 \\
\hline AF146523 & Malat1 & Metastasis associated lung adenocarcinoma transcript 1 & -7.8 \\
\hline NM_008587 & Mertk & C-mer proto-oncogene tyrosine kinase & -8.1 \\
\hline NM_008606 & Mmp11 & Matrix metallopeptidase 11 & -8.7 \\
\hline ВС015293 & Grem1 & Gremlin 1 & -15.8 \\
\hline NM_030888 & C1qtnf3 & $\mathrm{C} 1 \mathrm{q}$ and tumor necrosis factor related protein 3 & -31.5 \\
\hline
\end{tabular}


Table II. Gene expression changes between metastatic SCC in young and old mice (1235 genes).

\begin{tabular}{|c|c|c|c|}
\hline Accession & Symbol & Gene name & Fold change \\
\hline K02108 & Krt6a & Keratin $6 a$ & 1173.9 \\
\hline ВC011074 & Krt14 & Keratin 14 & 681.9 \\
\hline NM_011468 & Sprr2a & Small proline-rich protein $2 \mathrm{~A}$ & 601.4 \\
\hline AA7988563 & Krt17 & Keratin 17 & 558.6 \\
\hline NM_009265 & Sprr1b & Small proline-rich protein $1 \mathrm{~B}$ & 557.2 \\
\hline NM_011472 & Sprr2f & Small proline-rich protein $2 \mathrm{~F}$ & 526.6 \\
\hline NM_010357 & Gsta4 & Glutathione S-transferase, $\alpha 4$ & 461.6 \\
\hline ВC018397 & Crabp2 & Cellular retinoic acid binding protein II & 377.0 \\
\hline AV007306 & Krtdap & Keratinocyte differentiation associated protein & 320.2 \\
\hline NM_008470 & Krt16 & Keratin 16 & 293.0 \\
\hline NM_019645 & Pkp1 & Plakophilin 1 & 281.1 \\
\hline AV297961 & Dsp & Desmoplakin & 221.2 \\
\hline BC006780 & Krt5 & Keratin 5 & 169.6 \\
\hline AV229522 & Dsg3 & Desmoglein 3 & 163.7 \\
\hline AV241297 & Spink5 & Serine peptidase inhibitor, Kazal type 5 & 91.1 \\
\hline U88064 & Bnc1 & Basonuclin 1 & 87.1 \\
\hline NM_011641 & Trp63 & Transformation related protein 63 & 84.3 \\
\hline NM_008473 & Krt1 & Keratin 1 & 72.7 \\
\hline AI507307 & Sbsn & Suprabasin & 49.3 \\
\hline M21836 & Krt8 & Keratin 8 & 41.6 \\
\hline AV009441 & Ivl & Involucrin & 36.4 \\
\hline NM_009523 & Wnt4 & Wingless-related MMTV integration site 4 & 35.7 \\
\hline AV009267 & Foxq1 & Forkhead box Q1 & 22.4 \\
\hline NM_011415 & Snai2 & Snail homolog 2 (Drosophila) & 22.0 \\
\hline NM_008469 & Krt15 & Keratin 15 & 19.2 \\
\hline $\mathrm{X} 06340$ & Cdh3 & Cadherin 3 & 14.8 \\
\hline BB759833 & Foxc1 & Forkhead box $\mathrm{C} 1$ & 14.2 \\
\hline U34245 & Fosl1 & Fos-like antigen 1 & 13.8 \\
\hline J03458 & Flg & Filaggrin & 12.9 \\
\hline ВC008107 & Timp1 & Tissue inhibitor of metalloproteinase 1 & 11.5 \\
\hline AV359819 & Jag 1 & Jagged 1 & 10.8 \\
\hline NM_009704 & Areg & Amphiregulin & 10.6 \\
\hline NM_010664 & Krt18 & Keratin 18 & 10.0 \\
\hline ВB067079 & Wnt5a & Wingless-related MMTV integration site 5A & 9.0 \\
\hline ВC004663 & Dsc2 & Desmocollin 2 & 8.1 \\
\hline ВC003778 & Tcfap2c & Transcription factor AP- $2, \gamma$ & 8.1 \\
\hline AV264681 & Jag2 & Jagged 2 & 8.1 \\
\hline AV290079 & $\mathrm{Vdr}$ & Vitamin D receptor & 7.7 \\
\hline BB371406 & Fzd2 & Frizzled homolog 2 (Drosophila) & 5.5 \\
\hline NM_007669 & Cdkn1a & Cyclin-dependent kinase inhibitor 1A (p21) & 5.2 \\
\hline NM_009518 & Wnt10a & Wingless related MMTV integration site $10 \mathrm{a}$ & 5.1 \\
\hline NM_011691 & Vav1 & Vav 1 oncogene & -5.1 \\
\hline AI503808 & Ski & Sloan-Kettering viral oncogene homolog & -5.2 \\
\hline BG063053 & Traf3 & Tnf receptor-associated factor 3 & -5.3 \\
\hline AK004232 & Pla2g2d & Phospholipase A2, group IID & -5.4 \\
\hline BB472891 & Foxp1 & Forkhead box P1 & -5.6 \\
\hline U42467 & Lepr & Leptin receptor & -6.0 \\
\hline BB218245 & Traf1 & Tnf receptor-associated factor 1 & -6.0 \\
\hline BB376407 & Jarid1a & Jumonji, AT rich interactive domain $1 \mathrm{~A}$ (Rbp2 like) & -6.6 \\
\hline BB286270 & $\mathrm{E} 2 \mathrm{f5}$ & E2F transcription factor 5 & -7.0 \\
\hline BE284491 & Cflar & CASP8 and FADD-like apoptosis regulator & -7.2 \\
\hline BM293452 & Jarid2 & Jumonji, AT rich interactive domain 2 & -7.8 \\
\hline BB465968 & Tgfbr2 & Transforming growth factor, $\beta$ receptor II & -8.3 \\
\hline BB043576 & Ccnd3 & Cyclin D3 & -11.5 \\
\hline BB284583 & Itga4 & Integrin $\alpha 4$ & -12.1 \\
\hline AF146523 & Malat1 & Metastasis associated lung adenocarcinoma transcript 1 & -59.1 \\
\hline
\end{tabular}


Table III. Gene expression changes between primary and metastatic SCC in old mice (1038 genes).

\begin{tabular}{|c|c|c|c|}
\hline Accession & Symbol & Gene name & Fold change \\
\hline BB795191 & Mela & Melanoma antigen & 72.3 \\
\hline AK004668 & Tnfrsf $13 b$ & Tumor necrosis factor receptor superfamily, member $13 \mathrm{~b}$ & 24.0 \\
\hline M21836 & Krt8 & Keratin 8 & 19.3 \\
\hline NM_033374 & Dock2 & Dedicator of cyto-kinesis 2 & 17.0 \\
\hline NM_009288 & Stk10 & Serine/threonine kinase 10 & 9.6 \\
\hline BM124366 & Lepr & Leptin receptor & 8.8 \\
\hline NM_009515 & Was & Wiskott-Aldrich syndrome homolog (human) & 8.5 \\
\hline AV233043 & Runx3 & Runt related transcription factor 3 & 8.1 \\
\hline BI111620 & Timp3 & Tissue inhibitor of metalloproteinase 3 & 7.7 \\
\hline AA185884 & $\operatorname{Pax} 5$ & Paired box gene 5 & 6.8 \\
\hline NM_013566 & $\operatorname{Itgb7}$ & Integrin $\beta 7$ & 6.5 \\
\hline ВC018416 & Serpina10 & Serine (or cysteine) peptidase inhibitor, clade A, member 10 & 6.4 \\
\hline AV173139 & Stk17b & Serine/threonine kinase 17b (apoptosis-inducing) & 5.9 \\
\hline AV084904 & Ccl6 & Chemokine (C-C motif) ligand 6 & 5.8 \\
\hline NM_008404 & Itgb2 & Integrin $\beta 2$ & 5.8 \\
\hline AV230647 & Pik3r5 & Phosphoinositide-3-kinase, regulatory subunit 5, p101 & 5.8 \\
\hline NM_133949 & Ptov 1 & Prostate tumor overexpressed gene 1 & 5.8 \\
\hline AW214029 & Stat1 & Signal transducer and activator of transcription 1 & 5.5 \\
\hline NM_011476 & Sprr $2 \mathrm{j}$ & Small proline-rich protein $2 \mathrm{~J}$ & -5.0 \\
\hline ВC003778 & Tcfap2c & Transcription factor AP-2, $\gamma$ & -5.4 \\
\hline NM_009502 & $\mathrm{Vcl}$ & Vinculin & -5.4 \\
\hline AF083876 & Emp2 & Epithelial membrane protein 2 & -5.5 \\
\hline BE956701 & Nrip1 & Nuclear receptor interacting protein 1 & -5.5 \\
\hline NM_021878 & Jarid2 & Jumonji, AT rich interactive domain 2 & -5.6 \\
\hline U34245 & Fosl1 & Fos-like antigen 1 & -5.6 \\
\hline BI660199 & Rora & RAR-related orphan receptor $\alpha$ & -5.8 \\
\hline NM_015789 & Dkkl1 & Dickkopf-like 1 & -5.8 \\
\hline NM_008857 & Prkci & Protein kinase $\mathrm{C}, \mathrm{\iota}$ & -6.0 \\
\hline AV241297 & Spink5 & Serine peptidase inhibitor, Kazal type 5 & -6.0 \\
\hline NM_011146 & Pparg & Peroxisome proliferator activated receptor $\gamma$ & -6.1 \\
\hline AK009196 & $\operatorname{Tgm} 5$ & Transglutaminase 5 & -6.1 \\
\hline NM_011477 & Sprr2k & Small proline-rich protein $2 \mathrm{~K}$ & -6.1 \\
\hline NM_010207 & Fgfr2 & Fibroblast growth factor receptor 2 & -6.3 \\
\hline NM_008473 & Krt1 & Keratin 1 & -6.4 \\
\hline BC027242 & Vav3 & Vav 3 oncogene & 6.9 \\
\hline AK008979 & Cnfn & Cornifelin & -7.0 \\
\hline AI507307 & Sbsn & Suprabasin & -7.0 \\
\hline AF126834 & Ppl & Periplakin & -8.0 \\
\hline AF075434 & Trp63 & Transformation related protein 63 & -8.2 \\
\hline AV253195 & Dsg1b & Desmoglein $1 B$ & -9.1 \\
\hline NM_053087 & Epgn & Epithelial mitogen & -9.8 \\
\hline NM_007950 & Ereg & Epiregulin & -9.9 \\
\hline NM_013505 & Dsc2 & Desmocollin 2 & -10.0 \\
\hline NM_009704 & Areg & Amphiregulin & -10.1 \\
\hline AK014360 & Krt10 & Keratin 10 & -16.6 \\
\hline J03458 & Flg & Filaggrin & -16.9 \\
\hline BB269715 & Hif1a & Hypoxia inducible factor $1, \alpha$ subunit & -17.3 \\
\hline ВС026631 & Dsp & Desmoplakin & -22.7 \\
\hline Y11169 & Dsc3 & Desmocollin 3 & -32.0 \\
\hline BB151286 & Dsgla & Desmoglein $1 \alpha$ & -47.6 \\
\hline NM_009374 & $\operatorname{Tgm} 3$ & Transglutaminase 3 , E polypeptide & -49.5 \\
\hline NM_026335 & Sprrl9 & Small proline rich-like 9 & -53.6 \\
\hline NM_013504 & Dsc1 & Desmocollin 1 & -54.5 \\
\hline AI036317 & Lor & Loricrin & -107.2 \\
\hline
\end{tabular}


primary and metastatic tumors in mice which began the tumor induction protocol at 1 month old (young) and 1 year old (chronologically aged). As shown in Table I, comparison of primary tumors in young and chronologically aged mice revealed 458 differentially expressed genes. Increased expression of differentiation and adhesion genes was noted: small proline rich protein 3, 31.0-fold; keratin 4, 13.2-fold; desmocollin 1, 7.6-fold; desmocollin 2, 7.5-fold; transglutaminase 3, 5.1-fold. Genes regulating metastasis were differentially regulated in primary tumors from chronologically aged mice (serine peptidase inhibitor clade A, member 9, 5.9-fold; serine peptidase inhibitor clade $\mathrm{B}$, member 12, 5.7-fold; matrix metalloproteinase 9, -5.6-fold; matrix metallopeptidase 13, -6.6-fold; metastasis associated lung adenocarcinoma transcript 1,-7.8-fold; matrix metallopeptidase 11, -8.7-fold). Growth factor receptor expression was inhibited in primary tumors from chronologically aged mice (platelet derived growth factor receptor, -6.0-fold; fibroblast growth factor receptor 1, -5.7-fold; Jagged 1, -5.6-fold). These results indicate that primary tumors from chronologically aged mice were more highly differentiated with increased cellular adhesion and expressed decreased levels of growth factor receptors.

In contrast to the relative relatedness of primary tumors in young and chronologically aged mice, we detected substantially more differentially expressed genes between metastatic SCC in these animals (1235 genes, Table II). Metastatic tumors from chronologically aged mice were highly differentiated (keratin 6a, 1173.0-fold; keratin 14, 681.9-fold; small proline rich protein 2A, 601.4-fold; keratin 17, 558.6-fold; keratinocyte differentiation associated protein, 320.2-fold; keratin 16, 293.0-fold; keratin 5, 169.6-fold; keratin 1, 72.7-fold; suprabasin, 49.3-fold; keratin 8, 41.6-fold; involucrin, 36.4-fold; keratin 15, 19.2-fold; filaggrin, 12.9-fold; keratin 18, 10.0fold; vitamin D receptor, 7.7-fold). Genes regulating cellular adhesion were highly upregulated in metastatic HNSCC from chronologically aged mice (plakophilin 1, 281.1-fold; desmoplakin, 221.2-fold; desmoglein 3, 163.7-fold; cadherin 3, 14.8-fold; desmocollin 2, 8.1-fold). Genes regulating metastasis were differentially expressed in metastatic HNSCC from chronologically aged mice (serine peptidase inhibitor, 91.1-fold; snail homolog 2, 22.0-fold; tissue inhibitor of metalloproteinase 1, 11.5-fold; metastasis associated lung adenocarcinoma transcript 1, -59.1-fold). Growth factor signaling was differentially regulated in metastatic SCC from chronologically aged mice (wingless related MMTV integration site 4, 35.7-fold; Jagged 1, 10.8-fold; wingless related MMTV integration site 5A, 9.0-fold; Jagged 2, 8.1-fold; frizzled homolog 2, 5.5-fold; wingless related MMTV integration site 10a, 5.1-fold; leptin receptor -6.0-fold; transforming growth factor $\beta$ receptor II, -8.3-fold). Genes regulating cell proliferation were differentially expressed in metastatic HNSCC from chronologically aged mice (cyclin dependent kinase inhibitor 1A, 5.2-fold; cyclin D3, -11.5-fold). Proto-oncogene expression was decreased in metastatic HNSCC from chronologically aged mice (Vav1 oncogene, -5.1-fold; SloanKettering viral oncogene homolog, -5.2-fold). The Forkhead family of transcription factors was differentially expressed in these tumors (Forkhead box Q1, 22.4-fold; Forkhead box C1, 14.2-fold; Forkhead box P1, -5.6-fold). These results indicate that metastatic tumors in chronologically aged mice were more differentiated with increased cellular adhesion and less proliferative than their counterparts from young mice.

We compared primary and metastatic SCC in chronologically aged mice and identified 1038 differentially expressed genes (Table III). Metastatic SCC was substantially less differentiated compared to primary tumors in these mice (epithelial membrane protein 2, -5.5-fold; transglutaminase 5, -6.1-fold; keratin 1, -6.4-fold; cornifelin, -7.0-fold; suprabasin, -7.0-fold; keratin 10, -16.6-fold; filaggrin, -16.9-fold; transglutaminase 3,-49.5-fold; small proline rich like 9, -53.6-fold; loricin, -107.2-fold). Cellular adhesion genes also were downregulated in metastatic SCC compared to primary tumors (periplakin, -8.0-fold; desmoglein 1 B, -9.1-fold; desmocollin 2, -10.0-fold; desmoplakin, -22.7-fold; desmocollin 3, -32.0-fold; desmoglein $1 \alpha,-47.6$-fold; desmocollin 1, -54.5fold). Growth factors and receptors were downregulated in metastatic compared to primary SCC in chronologically aged mice (fibroblast growth factor receptor 2, -6.3-fold; epithelial mitogen, -9.8-fold; epiregulin, -9.9-fold; amphiregulin, -10.1fold). Genes involved in cell signaling pathways were upregulated (serine/threonine kinase 10, 9.6-fold; leptin receptor, 8.8-fold; phosphoinositide 3-kinase, 5.8-fold; signal transducer and activator of transcription 1, 5.5-fold). Genes regulating metastasis and migration were differentially regulated in metastatic HNSCC from chronologically aged mice (dedicator of cytokinesis, 17.0-fold; tissue inhibitor of metalloproteinase 3, 7.7-fold; serine peptidase inhibitor clade A member 10, 6.4-fold; vinculin, -5.4-fold). These results indicate that metastatic tumors in chronologically aged mice are less differentiated and demonstrate less cellular adhesion and increased intracellular signaling than corresponding primary tumors.

\section{Discussion}

Despite association of diminished DNA repair with premature aging, we did not observe decreased expression of DNA repair genes in tumor tissue from chronologically aged mice. These results suggest that accumulation of low level DNA damage during the first year of mouse lifespan may predispose the animals to primary HNSCC development during chemical carcinogenesis. Additionally, HNSCC induced in 1 year old mice was poorly vascularized and developed areas of necrosis, which may reduce the number of metastatic clones. The number of metastatic cervical lymph nodes in these chronologically aged mice was statistically lower than other groups. Metastatic tumors in cervical lymph nodes also showed areas of necrosis. Neither primary nor metastatic tumors from the different groups of mice expressed lower levels of angiogenic factors such as vascular endothelial growth factor. These results suggest that capillary endothelium in chronologically aged mice may be less responsive to angiogenic factors resulting in decreased tumor vascularization and increased cell death. Previous studies have demonstrated decreased angiogenic response in aged organisms.

A number of transcription factors were differentially regulated in the aging model of HNSCC. The TWIST gene which has been shown to enhance metastasis in many types of cancer was downregulated in primary HNSCC in chrono- 
logically aged mice $(21,22)$. The Runx 3 transcription factor is a major tumor suppressor in many tumor types including human HNSCC where high expression is correlated with better prognosis $(23,24)$. p63 which is required for proliferation and differentiation of epithelial cells was dramatically upregulated in tumors from chronologically aged mice (25). The FoxP1 forkhead transcription factor is downregulated in multiple cancer types (26), and its expression also was decreased in metastatic tumors from chronologically aged mice. The oncogenic transcription factor PAX5 is upregulated in human HNSCC (27), and its expression also was increased in metastatic tumors in chronologically aged mice. The Jumonji domain containing transcriptional repressors Jarid1a and Jarid2 have potential tumor suppressor function (28), and their expression is inhibited in tumors from chronologically aged mice. The basonuclin transcription factor is markedly upregulated in basal cell carcinoma in epidermis (29), and also in metastatic tumor cells from chronologically aged mice. Elements of the Wnt signaling pathway were altered in HNSCC such as upregulation of Wnt4, Wnt10a, and frizzled homolog 2 in metastatic tumors from chronologically aged mice. Members of the nuclear hormone receptor family such as peroxisome proliferator activated receptor $\gamma$ was upregulated in primary SCC in chronologically aged mice, but downregulated in metastatic tumors from chronologically aged mice. These results indicate that HNSCC in our mouse model recapitulates many of the alterations in transcription factor gene expression found in human cancer.

\section{Acknowledgements}

We thank Dr T. Triche, B. Schaub, and S. Waidyaratne (Genomics Core Facility, Children's Hospital, Los Angeles, CA) for assistance with microarray analysis. This study was supported by National Institutes of Health grant DE14283 to DLC.

\section{References}

1. Balducci L and Ershler WB: Cancer and aging: a nexus at several levels. Nat Rev Cancer 5: 655-662, 2005.

2. Campisi J: Senescent cells, tumor suppression, and organisma aging: good citizens, bad neighbors. Cell 120: 513-522, 2005.

3. Anisimov VN, Sikora E and Pawelec G: Relationships between cancer and aging: a multilevel approach. Biogerontology 10 323-338, 2009.

4. Von Wagenheim KH and Peterson HP. Control of cell proliferation by progress in differentiation: clues to mechanisms of aging, cancer causation, and therapy. J Theor Biol 193: 663-678, 1998

5. Reya T, Morrison SJ, Clarke MF and Weissman IL: Stem cells, cancer, and cancer stem cells. Nature 414: 105-111, 2001.

6. Tomatis L: Cancer: causes, occurrence, and control. IARC Sci Publ 100. IARC, Lyon, 1990.

7. Derhovanessian E, Solana R, Larbi A and Pawelec G: Immunity, aging, and cancer. Immun Aging 15: 11, 2008.

8. Walter S, Boley G, Buhring HJ, Koch S, Wernet D, Zippelius A, Pawelec G, Romero P, Stevanovic S, Rammensee HG and Gouttefangeas C: High frequencies of functionally impaired cytokeratin 18 specific $\mathrm{CD}^{+} \mathrm{T}$ cells in healthy HLA-A2+ donors. Eur J Immunol 35: 2876-2885, 2005.

9. Ku TKS and Crowe DL: Impaired T lymphocyte function increases tumorigenicity and decreases tumor latency in a mouse model of head and neck cancer. Int J Oncol 35: 1211-1221, 2009.
10. Mikhnin AE, Barchuk AS and Wagner RI: Kinetics of visual growth of skin melanoma. Russ Oncol J 2: 29-32, 2004.

11. Narita M and Lowe SW: Senescence comes of age. Nat Med 11: 920-922, 2005.

12. Sliwinska MA, Mosieniak G, Wolanin K, Babik A, Piwocka K, Magalska A, Szczepanowska J, Fronk J and Sikora E: Induction of senescence with doxorubicin leads to increased genomic instability of HCT116 cells. Mech Aging Dev 130: 24-32, 2009.

13. Jackson JG and Pereira-Smith OM: Primary and compensatory roles for $\mathrm{Rb}$ family members at cell cycle gene promoters that are deacetylated and downregulated in doxorubicin induced senescence of breast cancer cells. Mol Cell Biol 26: 2501-2510, 2006.

14. te Poele RH, Okorokov AL, Jardine L, Cummings J and Joel SP: DNA damage is able to induce senescence in tumor cells in vitro and in vivo. Cancer Res 62: 1876-1883, 2002.

15. Roberson RS, Kussick SJ, Vallieres E, Chen SY and Wu DY: Escape from therapy induced accelerated cellular senescence in p53 null lung cancer cells and in human lung cancers. Cancer Res 65: 2795-2803, 2005.

16. Berns A: Senescence: a companion in chemotherapy? Cancer Cell 1: 309-311, 2002

17. Rebbaa A, Zheng X, Chu F and Mirkin BL: The role of histone acetylation versus DNA damage in drug induced senescence and apoptosis. Cell Death Differ 13: 1960-1967, 2006.

18. Storchova Z and Pellman D: From polyploidy to aneuploidy, genome instability, and cancer. Nat Rev Mol Cell Biol 5: 45-54, 2004.

19. Puig PE, Guilly MN, Bouchot A, Droin N, Cathelin D, Bouyer F, Favier L, Ghiringhelli F, Kroemer G, Solary E, Martin F and Chauffert B: Tumor cells can escape DNA damaging cisplatin through DNA endoreduplication and reversible polyploidy. Cell Biol Int 32: 1031-1043, 2008.

20. Ku TKS, Nguyen DC, Karaman M, Gill P, Hacia JG and Crowe DL: Loss of p53 expression correlates with metastatic phenotype and transcriptional profile in a new mouse model of head and neck cancer. Mol Cancer Res 5: 351-362, 2007.

21. Yang MH, Wu MZ, Chiou SH, Chen PM, Chang SY, Liu CJ, Teng SC and Wu KJ: Direct regulation of TWIST by HIF-1 $\alpha$ promotes metastasis. Nat Cell Biol 10: 295-305, 2008.

22. Satoh K, Hamada S, Kimura K, Kanno A, Hirota M, Umino J, Fujibuchi W, Masamune A, Tanaka N, Miura K, et al: Upregulation of MSX2 enhances the malignant phenotype and is associated with twist 1 expression in human pancreatic cancer cells. Am J Pathol 172: 926-939, 2008.

23. Tanji Y, Osaki M, Nagahama Y, Kodani I, Ryoke K and Ito H: Runt related transcription factor 3 expression in human oral squamous cell carcinomas: implicatioin for tumor progression and prognosis. Oral Oncol 43: 88-94, 2007.

24. Ito Y: RUNX genes in development and cancer: regulation of viral gene expression and the discovery of RUNX family genes. Adv Cancer Res 99: 33-76, 2008.

25. Koster MI, Kim S, Mills AA, DeMayo FJ and Roop DR: p63 is the molecular switch for initiation of an epithelial stratification program. Genes Dev 18: 126-131, 2004.

26. Giatromanolaki A, Koukourakis MI, Sivridis E, Gatter KC, Harris AL and Banham AH: Loss of expression and nuclear/ cytoplasmic localization of the FOXP1 forkhead transcription factor are common events in early endometrial cancer: relationship with estrogen receptors in HIF-1 $\alpha$ expression. Mod Pathol 19: 9-16, 2006.

27. Norhany S, Kouzu Y, Uzawa K, Hayama M, Higo M, Koike H, Kasamatu A and Tanzawa H: Overexpression of PAX5 in oral carcinogenesis. Oncol Rep 16: 1003-1008, 2006.

28. Wolf SS, Patchev VK and Obendorf M: A novel variant of the putative demethylase gene, s-JMJD1C, is a coactivator of the AR. Arch Biochem Biophys 460: 56-66, 2007.

29. Cui C, Elsam T, Tian Q, Seykora JT, Grachtchouk M, Dlugosz A and Tseng H: Gli proteins upregulate the expression of basonuclin in basal cell carcinoma. Cancer Res 64: 5651-5658, 2004. 